\title{
The Effect of Anomalous Magnetic Fields on Malignant Diseases
}

\author{
Trifunovic Nikola1, Cizmic Vladislav² \\ ${ }^{1}$ Retired Geophysicist, Geoinstitut, Belgrade, Serbia \\ ${ }^{2}$ Doctor of Dentistry, Belgrade, Serbia \\ Email: ntrifunovic41@yahoo.com
}

Received 5 April 2015; accepted 22 April 2015; published 28 April 2015

Copyright (C) 2015 by authors and OALib.

This work is licensed under the Creative Commons Attribution International License (CC BY). http://creativecommons.org/licenses/by/4.0/

(c) (i) Open Access

\begin{abstract}
Introduction: Natural earth's magnetic field (EMF) is promoter of mother cell division into two daughter cells. Cancer is a cell that divides unlimited and destroys the body. Aim: Measurements presented in this paper show that malignant diseases are formed in anomalous magnetic fields (AMF) in patient's beds. Removal of patients from AMF explains the etiological uncertainties in the medical literature. Methods: Measuring of AMF in patients' beds was performed by proton magnetometer. Measuring points are on square net, $10 \times 10 \mathrm{~cm}$. The results are shown with contour lines on drawings. The health status of residence in the Earth's natural magnetic field (EMF) was monitored. The etiologic data from the literature are explained. Results: Research lasted 25 years. AMF were measured in 1500 patients' beds. There was a correlation between AMF and location of the diseased organ. AMF surfaces were shown in beds where the diseased organ is, on the contrary, there were natural values of EMF in the rest of the bed surface. Furthermore, the results found that three cases of breast cancer with metastases survived after surgical interventions and subsequent removal into natural EMF in contrast to 26 cases with the same diagnosis and surgical interventions who died after short period and staying in AMF. Another example was brain malignancy, which was decreased by living in natural EMF for one year. Conclusion: Based on the results of measurement of AMF, the medical condition of the patient when staying in a natural EMF, and explanation of uncertainties etiologies, the main conclusion was: AMF is the main cause of the malignant diseases.
\end{abstract}

\section{Keywords}

Earth's Magnetic Field, Anomalous Magnetic Field, Malignant Disease and Metastases

Subject Areas: Public Health

\section{Introduction}

After facing MD in family and talking with doctors it is learnt that there are three main cancer causes: viral, che-

How to cite this paper: Nikola, T. and Vladislav, C. (2015) The Effect of Anomalous Magnetic Fields on Malignant Diseases. Open Access Library Journal, 2: e1459. http://dx.doi.org/10.4236/oalib.1101459 
mical (cancerogenous substances) and physical. Doctors' comment is: we know a lot about viral and chemical agents, but about physical it is not known much. This has made us start geophysical measurements of physical fields in the places where people suffering from cancer, cardio-vascular disease, diabetes and mental disturbances live. The measurements have lasted for more than 25 years. Physical fields are, also, measured in the places where people suffering from some other diseases live, but not so extensively. It has immediately been found that people suffering from cancer sleep in beds with characteristic EMF values, of much higher intensities than the natural EMF, called AMF, or lower, anomalous zones (Az). At first, radioactive radiation was measured and it was always within the scope of natural quantities, so there was no need to continue measuring it. Pathology professor at the Pathology Institute, has enabled us to observe a microscopic cancer preparation. This looked like microscopic serpentine preparation (a very magnetic ultrabasic rock), containing bits of magnetite as phenocrysts. This was a stimulation for even more extensive cancer cause investigation. Simultaneously with insitu measurements, we studied biological and medical literature, particularly regarding etiology and pathology. It is understood that cancer is a cell gone wild dividing itself endlessly and destroying an organ and organism, consequently. But we realize that biological science does not know what a mother cell into two baby cells division promotor is. Soon, the paper "Breathing Enables the Magnetic Properties of Erythrocytes (Hem Fe) Oxygen, Cells and Carbondioxid" [1] gets published, fully explaining: 1) a mother cell division into two baby cells; 2) malignant diseases genesis; 3) it has been found and proven that the magnetic characteristics of a person's breathing enable respiration, and the constant fallacy that diffusion and partial pressure enable respiration has been eliminated; 4) the nervous system functioning in respiration regulation has been clearly explained. It can freely be stated that this scientific work is fundamental for all further biology and humanistic researches. The aim of this work is: to prove, by measurements, that the malignant diseases are formed in AMF in the beds of diseased people; to move the diseased away from AMF and to observe their health conditions; to explain etiopathological obscurities from the medical literature by understanding the AMF and natural EMF; to present the results obtained in this paper. This paper: "The Effect of Anomalous Magnetic Fields on Malignant Diseases" is a particular addition of the malignant diseases genesis, theoretically presented in literature [1].

\section{Work Methods}

In the research, the attention has been paid to geomagnetic field. By measurements, EMF spatial changes have been discovered, i.e. the locations of AMF have been found. AMF, i.e. Az appears because of incorrect placement of magnetic things in living spaces. There are, also, variable magnetic fields, originating from the electromagnetic fields, characteristical for every Az.

Measurement techniques are geophysical instruments, and geophysical measurements and interpretation of the measured values performed.

People place things in the wrong way and, being unaware of it, surround themselves by the materials which are pollutants of the living spaces-magnetic and electro-magnetic "smog".

Measurements of AMF in the diseased people's beds was performed. Measuring points were on square net, 10 $\times 10$. The results are shown with contour lines on drawings. Prelocation of beds away from Az into the space with EMF took place. The health condition was observed during the stay in EMF. Etiological data from literature were explained.

\section{Measurement Results}

The total resulting intensity vector of the whole AMF was measured. The instrument was "protonic magnetometer", $100 \mathrm{nT}$ (nanoteslas) factory precision. The results obtained are presented graphically on sketches in the forms of iso-anomalies (lines with the same AMF quantities), given in microteslas $(\mu \mathrm{T})$.

On the sketches are shown $\mathrm{Az}$ on beds used for night and day rest by a person with malignant neoplasms (Neo), as well as the diseased ones with metastases (meta). Diagnoses are, mostly, given by the Institute for Oncology and Radiology, Belgrade. The disease classification is in accordance with the international classification (IDC-10) [2]. The medical therapy included therapy directives and surgical operations advised by a council. It should be pointed out that, for the good therapeutic results, the most important thing is to move the patient away from Az and place him/her in a natural EMF. 


\subsection{Sketches with Only One Az in Bed}

Sketch 1 shows Neo on cervix C 53.1. This sketch shows magnetic field status in a single bed. On the central part of the single bed an Az is found (over $10 \mu \mathrm{T}$ ) covering the bed's user genital region. Az has caused Neo on cervix appearance. The $\mathrm{Az}$ and the location pathological changes connection is obvious. This bed user is moved to another bed with no Az. The woman, using the bed with no Az for over 20 years, is completely healthy. An interesting thing happened to her husband, who ignored the findings. He moved to sleep in the wife's first bed shown in the sketch 1 with Az and, after six months he got leg joint atherosclerosis and the beginning of gangrene on his left leg. Advice by one of this paper's author (TN), to place the bed into another location. After medical intervention, he gets healthy.

\section{SKETCH WITH MEASUREMENT RESULTS}

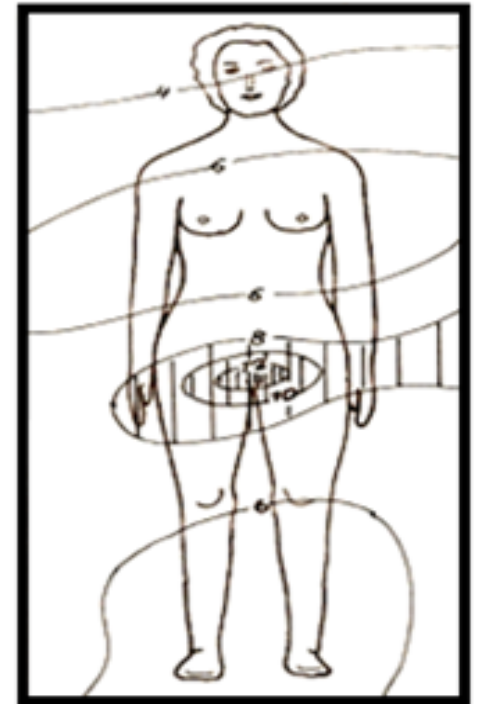

Sk.1 Neo on cervix C 53.1

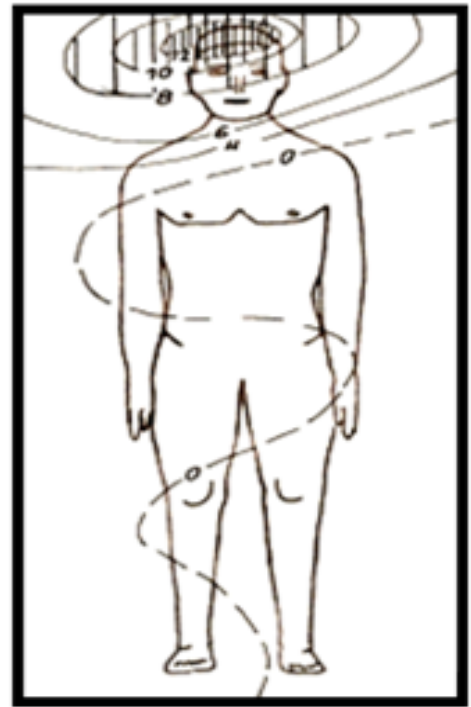

Sk.2 Neo on CNS C 71.2

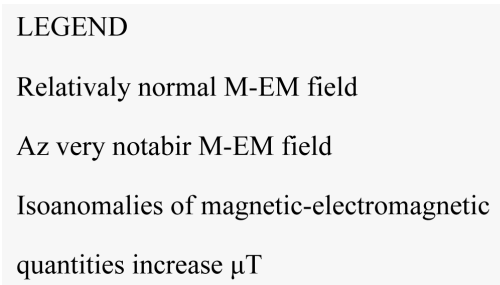

Sketch 2 shows Neo on CNS C 71.2. This single bed's space is mostly undisturbed because the quantities were, mostly, of zero increase. One Az was found in the upper central bed's part. The bed user's forehead entered a distinct Az. After two years from sleeping in this space, he had got brain Neo. The connection was fully confirmed. Suggestion was given to what should the diseased do to use the space with no Az.

Sketch 3 shows Neo on CNS Glioblastom multiforme IV. This single bed space was, mostly, undisturbed because in the most part of it a natural magnetic field was found, except in the upper central part with a distinct $\mathrm{Az}$, over $10 \mu \mathrm{T}$, causing Neo on CNS. The person was moved away and placed in an area with no Az. The connection between $\mathrm{Az}$ and the disease was obvious. After surgical operation, relapse with dimension appeared as shown in CT record in Figure 1. After that, the person was moved away from the Az. Moreover, Figure 2 shows the Neo CT record after one year of staying in the natural EMF (Figure 2). For five years there were neither relapses nor meta. Another three patients had this Neo type at the same time and they were all treated with the same therapy. However, two years later, three patients died and only the patient who, after relapses, was moved to spend her day and night rest in the natural EMF, survived. 
SKETCH WITH MEASUREMENT RESULTS

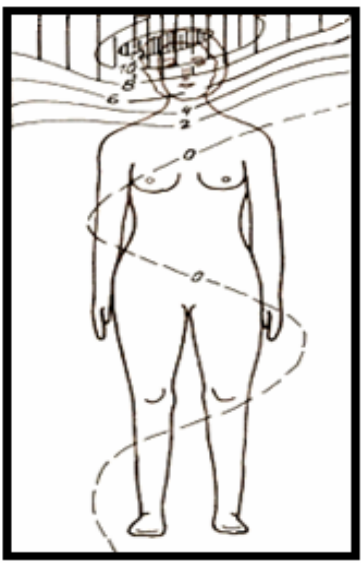

Sk.3 Neo on CNS

Glioblastom multiforme IV

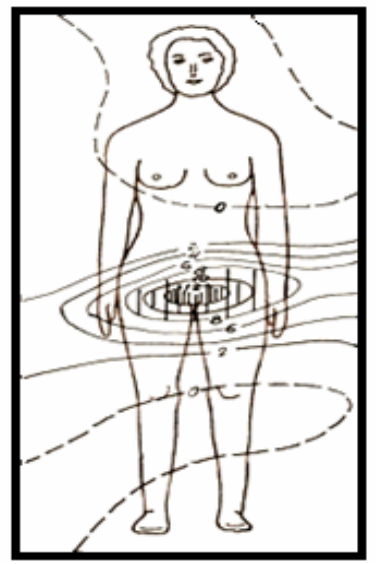

Sk.4. Neo on genitals C 53.1

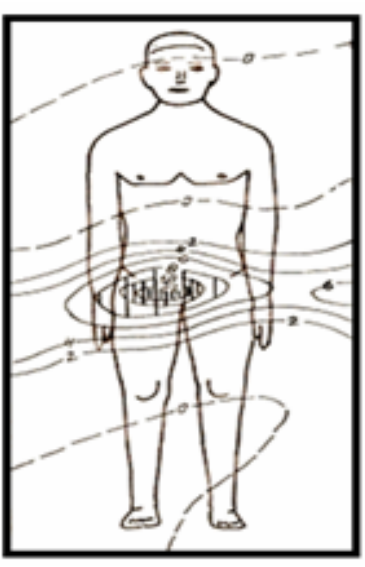

Sk.5. Neo on colon C 18.79

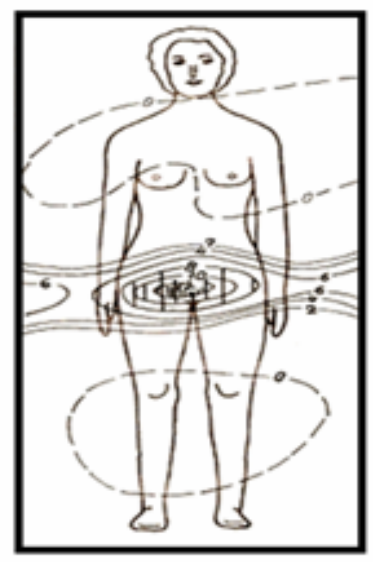

Sk.6 Neo on genitals C 53.1

\section{LEGEND}

Relativaly normal M-EM field

Az very notabir M-EM field

Isoanomalies of magnetic-electromagnetic

quantities increase $\mu \mathrm{T}$

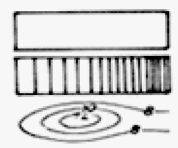

Sketch 4 shows Neo on genitals C 53.1. This bed space was, mostly, undisturbed. An outstanding Az was registered in the central bed's part where the bed user's genitals were, and she got Neo on genitals. Connection was confirmed here. The user was warned to eliminate the Az cause, which was, probably, done.

Sketches 5 and 6 show Neo on colon-C 18.79 and Neo on genitals-C 53.1. These single beds spaces were mostly undisturbed because of no Az were found with the natural EMF in their most parts. The central beds' parts were covered with $\mathrm{Az}$ above $10 \mu \mathrm{T}$, where colon was placed (Sketch 5) and genitals (Sketch 6). Connection between the Neo appeared and Az was confirmed.

CT records CNS

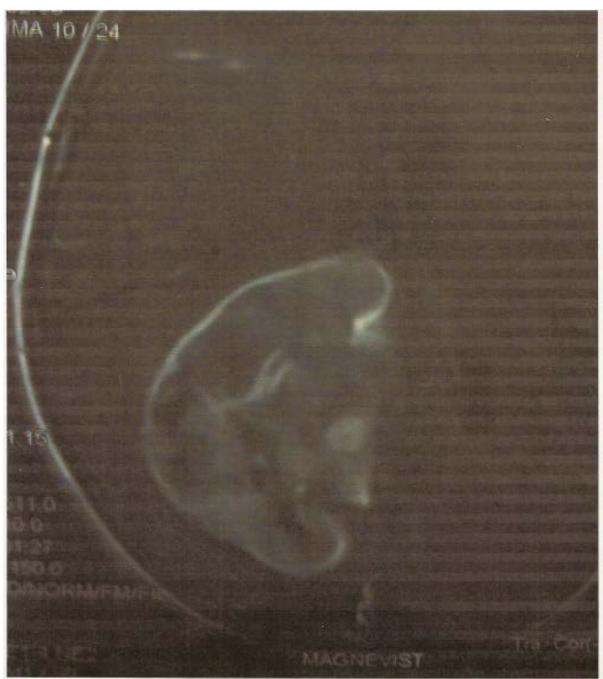

Figure 1. Relaps with dimension as shown in CT record. 


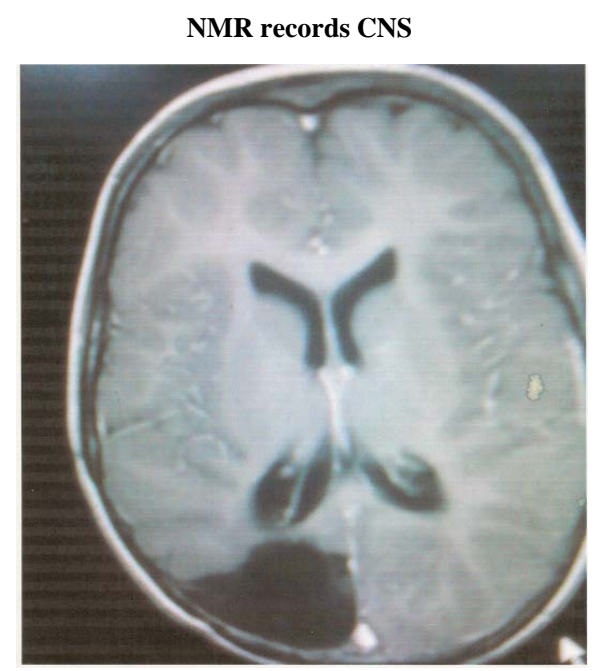

Figure 2. The Neo NMR record after one year of staying in the natural EMF.

The common thing for the presented Sketches 1, 2, 3, 4, 5 and 6, was that Az was located only on the body parts where diseases appeared, which is a clear in-situ proof that the external surrounding Az is the Neo cause. In addition, there were meta appearances with none of the patients from the above Sketches. This points that the remaining part of beds have no Az formed which could, firstly, create a body viscous magnetization (BVM) in the beds users [3] [4], being a condition for Neo appearance. It could be concluded that with no BVM there are neither diseases nor meta. BVM occurs when a body part is placed in AMF, i.e. Az.

\subsection{Sketches with Neo and Metastases}

Sketch 7 shows Neo on breast with meta C 50.0. This bed space was extremely disturbed and was characterised by high AMF over entire bed. A distinct Az was registered in the part where, during the night rest and the right breast which was diseased. Here, metastases were found on armpit lymph nodes. Connection between $\mathrm{Az}$ and the disease was obvious. This bed user had, immediately, moved into a space with no Az. After a few years, scintigraphy registered accumulation of radioactive markers in head's region. The cause was discovered and it was metal hairpins, which after not using them for two months a medical check up showed that the health state was normal. After some time, scintigraphy discovered radioactive accumulation in shoulder region. The cause was found and removed. After two months, the medical check up, after two months, showed that everything was normal again. After this, the person is alive and healthy for more than 15 years, after this. On the contrary, nine of her hospital room mates suffering from the same disease (Neo on breast and meta) all died after three years. The survived woman's comment was: "I am lucky because after measurements taken, the Az was eliminated from my bed."

Sketch 8 shows Neo on breast with meta C 50.0. This bed area was largely covered with a higher AMF. A high Az was registered in the area of the bed user's breast during night and day rest. After a few years of sleeping there the bed user got Neo on breast, metastases were found on armpit lymph nodes. Connection between $\mathrm{Az}$ and the disease was clear. During the person's medical treatment doctor strictly requested elimination of Neo only in the breast area, no other therapy was accepted. Chemotherapy, radiotherapy and hormone therapy were rejected. Measurements at her home and weekend cottage were taken place. She was informed in the kind of space she should spend her day and night rest, what she accepted immediately and started to use the space with no Az. For more than 10 years she is alive, oncologists are surprised. She said that her hospital room mates with the same diagnosis and surgical operation, died very soon after it. They were treated by the breast surgical radical operation followed by chemotherapy. Only one of nine of them survived because she was moved away from Az.

Sketch 9 shows Neo on breast with meta C 50.0. AMF measurements took place in 1995. Connection between $\mathrm{Az}$ and Neo location was confirmed. There were eight cases, only one woman survived and is completely healthy. Her comment was: "I am fully aware that moving into the healthy area saved my life." 
SKETCH WITH MEASUREMENT RESULTS

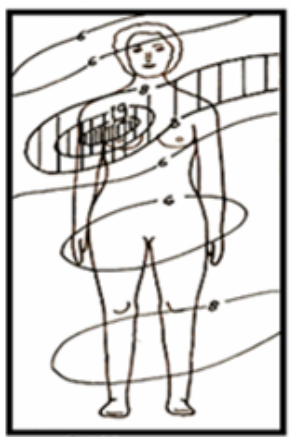

Sk.7 Neo on breast with meta C 50.0

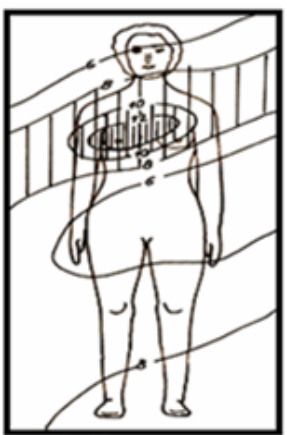

Sk 8. Neo on breast with meta C 50.0

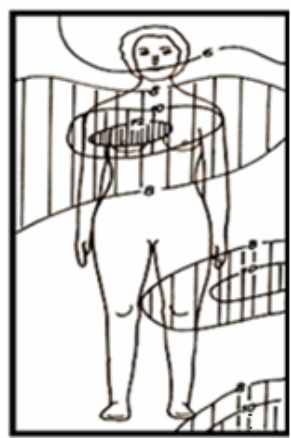

Sk 9. Neo on breast with meta C 50.0

\footnotetext{
LEGEND

Relativaly normal M-EM field

Az very notabir M-EM field

Isoanomalies of magnetic-electromagnetic

quantities increase $\mu \mathrm{T}$
}

\subsection{Sketches with Tumour (It Is Not Known Whether They Are Malignant) Where Recovery Occurs with No Medical Intervention}

Sketch 10 shows Tu on head, Sketch 11 shows Tu on prostate and Sketch 12 shows Tu on breast. Connection between Tu on head, prostate, breast and $\mathrm{Az}$ was clear. There were three patients who refused all medical help except elimination of Az from living spaces, which took place. The disease withdrawn, i.e. Tu cells in the natural EMF regenerated with all the three diseased. Probably, the diseases were in the starting phase, so there was no permanent cells magnetisation. More than 15 years have passed from the tumour appearance.

There is documentation about a large number of measured beds used by people with Neo. In all the beds connection between $\mathrm{Az}$ and Neo was confirmed. Presentation of a large number of sketches is unnecessary. After 25 years of EMF measurements performed in the cancer patients' beds (there were over 1500) no disease created in the natural EMF has been found.

SKETCH WITH MEASUREMENT RESULTS

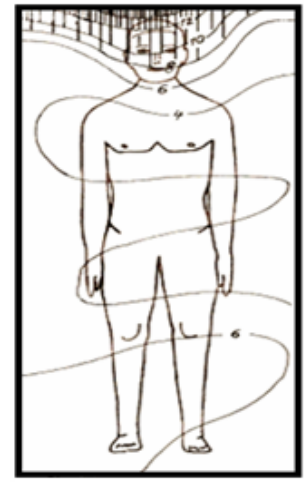

Sk-10 Tumour on head

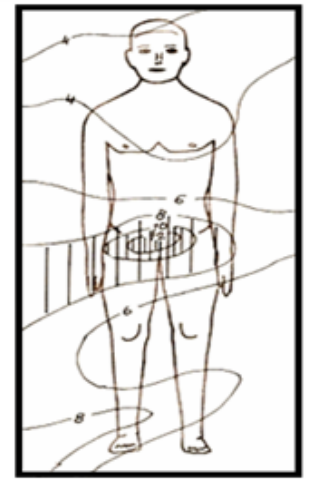

Sk.11 Tumour on prostate

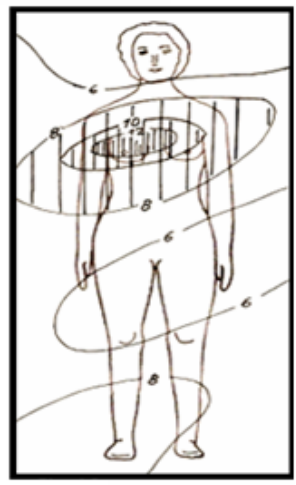

Sk.12 Tumour on breast

LEGEND

Relativaly normal M-EM field

Az very notabir M-EM field

Isoanomalies of magnetic-electromagnetic

quantities increase $\mu \mathrm{T}$

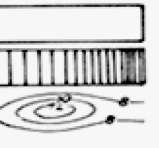




\section{Explanation of Etiology Obscurities, Found in Literature}

In the literature about Neo there are data which have not been explained. In the following text these unexplained subjects are explained

\subsection{Different Number of the Malignant and Cardiovascular Diseases (CVD)}

"Malignant tumours, after cardiovascular, are the most frequent death causes in the majority of the developed countries of the world" [3] [4]. Explanation of this different number of the diseased is in the frequency of AMF (more frequent) as a cause of CVD, and variable AMF as the Neo cause is less present in the people's living spaces.

\subsection{Cancer Appearance Results Obtained in Cities and Villages}

Cancer appearance results obtained in cities and villages (Graph 1) [5] clearly shows that city inhabitants are much more exposed to the variable AMF than the village ones. The village inhabitants lot of their time spend in the natural EMF, which is a great contribution to the smaller number of the diseased.

\subsection{Textile Machine Female Workers Found to Be More Frequently Victims of Right Breast Cancer Disease}

“Textile workers operating on machines were found to be more frequently victims of right breast cancer disease. This case has not been explained so far.” [5]. The workers operate on the machines mostly with their right hands and, after that, sleep on their right body side, so the right breast is more exposed to the AMF source, therefore, endangered.

\subsection{According to Lowseley and Kirwin (1979), Urothelium Tumours Are More Frequent on the Right SDE Ureter than on the Left Side Ureter}

“According to Lowseley and Kirwin urothelium tumours are more frequently found on the right side ureter than on the left side ureter" [6]. The explanation is as follows: right side kidney is located lower than the left side one, greater number of people sleep on the right body side and AMF decreases with the increase of the distance from its source, therefore, the right side kidney is closer to the contact bed-human's body. AMF vectors' direction is always towards the bed user's body. Now it is clear why there is more those who have the right kidney diseased than the left side one.

\subsection{During the Benzanthracen Methylation Cancerogenous Influence Increases}

"During the benzanthracen menthylation, cancerogenous influence increases" [7]. Since methyl $\left(\mathrm{CH}_{3}\right)$ is a molecule with paramagnetic characteristics, it is normal that with the increase of $\mathrm{CH}_{3}$ molecules number, additive substance magnetic characteristics increase, therefore, a logical conclusion is "cancerogenous influence" increases.

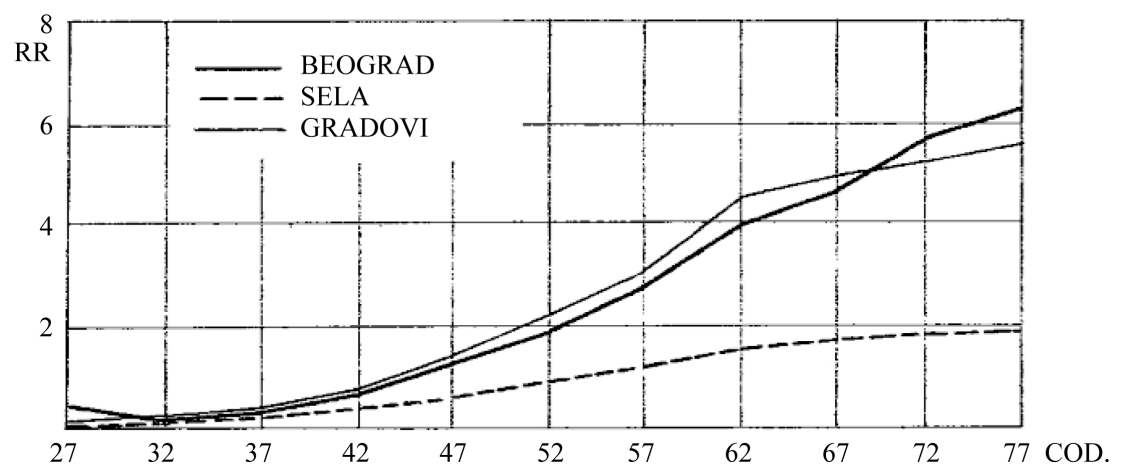

Graph 1. Cancer appearance results obtained in cities and villages [5]. Legend: Beograd-Belgrade; Sela_villages; Gradovi-cities. 


\subsection{Experimental Research on Animals}

Experimental research obtained on animals with implanted oncogenes has several, moderately said, surprising aspects. The first, most of the tumors appears are monoclonal, in spite the fact that each somatic cell bears identical transgenic oncogenic construction. The second, percentage of animals which get tumor is $20 \%-30 \%$, but why not all animals? The third, in many cases the latent period is unexpectedly long — but not much faster, while all the genetic predispositions are present all the time in each somatic cell?” [8]

The explanation is: The first, since AMF is a vector variable, and this vector is tied with a point and influences only upon one cell (most oftenly), it is absolutely clear why the occured tumours are mostly monoclonal. The second, only the animals spending most of their time in the areas contaminated with cancerogenous AMF (20\% - 30\%) get tumor, therefore, it is absolutely clear why the minor part of the transgenic animals get the disease. The third, areas with AMF, firstly, must build BVM of an organ or part of an organ where tumour will appear. This building needs time and that is why the latent period is long. More detailed explanation given in the 3.21 section, "large number of people spends time in AMF but not all get diseased".

\subsection{Nobel Prize in 1966}

Nobel prize given in 1966 for the work "Discovering the Virus which Causes Tumours... Those tiny subcellular organisms having RNA as their inherited basis." [9]

Interpretation of this mistake concerning the Neo appearance cause as follows: RNA is a protein with distinct paramagnetic characteristics because it is synthesized by the molecules which are paramagnetics [1]. Virus enters a host cell and starts multiplying. If the organ with viruses is in an anomalous magnetic field, then it supports the organ to form BVM. In such surrounding, a paraoncogen mutation is easily performed, consequently the cancer appearance. Viruses are only BVM appearance accelerators, but not the cancerogenous causes.

\subsection{Smoking and Cancer}

This subtitle may also mean cardiovascular and many other diseases are found with smokers by the official medical science, more oftenly than with non-smokers. The explanation why smokers more often get diseased, is in the paramagnetic characteristics of the tobacco smoke substances. In ref. [1] it is shown that each cell has its magnetisation and it is the strongest in the cell's nucleus, where chromosomes and mostly chromatine substances are. During the tobacco cells burning, the magnetised particles (from cells) enter lungs, blood and, through blood vessels, other organs with smoke. Since they have paramagnetic characteristics, they accumulate in the space with increased magnetic flux, what is logical. This is why the tobacco smoke particles, as paramagnetics, accelerate BVM creation in an organ or its part. This is a clear explanation why tobacco smoke is very harmful for many diseases which can be connected with AMF. Conclusion would be that tobacco smoke is harmful not as the Neo cause but as the BVM appearance accelerator. AMF from external surrounding is the main cause of diseases statistically connected with smoking.

\subsection{Malignant Cells Presence in Blood-Carcinemia-Is Frequent with Neo}

"Malignant cells presence in blood—carcinemia—is frequent with Neo. This, of course, still does not mean metastasis" [10]. In order that malignant cells perform colonisation (metastasis) it is necessary that AMF creates BVM in an organ. As the very malignant cells have magnetisation, colonisation can be found only in the tissue in which there must be BVM formed, and then metastases occur.

\subsection{Asbestos}

Officially, asbestos is a cancerous material. Chemically, asbestos is magnesium and divalent iron silicate, which makes it difficult to dissolve and has an increased susceptibility. These characteristics force asbestos to accumulate in the area under the AMF influence. Now it is clear why in the lungs tumour there are: microscopic asbestos "bodikins". These bodikins penetrate through to the cell nucleus membrane (where they stay unchanged) because the cell's magnetisation center is in nucleus [1]. Asbestos accumulation accelerates BVM in lungs and nothing else. 


\subsection{Radioactivity and Cancer}

Areas with radioactive accidents (nuclear station damage, atomic bomb etc.) show the appearance of the larger Neo number. The explanation is the following: An increased radioactive gamma radiation is present during the mentioned accidents (it should be noted that natural gamma radiation is manifoldly useful for the living world on Earth). Largely increased gamma radiation can cause radioactive damage to living beings similar to "burns". The increased number of malignant diseases is the result of DNA increased mutation because of the manifold increased gamma radiation. Elementary gamma radiation quanta by hitting, for example, hydrogen which, by weak chemical connections, ties nitrogenous bases to DNA scales, strikes it out from equilibrium and nitrogenous bases connections get weaker. If there is AMF where a person sleeps, the structural formula magnetic rotation on bases previously connected by hydrogen happens, and this is mutation. It is logical that paraoncogen can, on this occasion, transit into oncogen. The conclusion is that, when gamma radiation is manifold increased the genes on DNA mutation possibility is increased. BVM creation of the body part where AMF is present is, also, accelerated because all radioactive elements and some substances are paramagnetics.

\subsection{Proof That Malignant Cells Are Magnetized}

The proof that malignant cells are magnetized is nuclear magnetic resonance. In order to clearly notice the tissue with Neo, applications are injected into blood, which is a paramagnetic marker. In the most recent method cancer cells are caught by magnetic poles from blood flowing over the magnetic poles, after that they are sent to laboratory for further testing. Therefore, it is clear that cancer cells are magnetised because they were created in Az produced by external surrounding.

\subsection{Breast Tissue Density}

"Breast tissue density on mamography equal to or greater than $75 \%$, is connected to increased risk compared to mammography finding without increased density (RR 5.0.95\% CI 3.6 - 7.1); (16)” [11]. Breast tissue density greater than $75 \%$, is connected with more serious breast cancer risk. Nice proof that AMF from external surrounding are breast cancer causes is tissue density (breast cell). After a long time spent in AMF, breast density increases, which results from BVM appearance when substances with paramagnetic characteristics concentrate. Basic characteristics of magnetised substances are that inter-molecular distances get shorter which means density increase. Suggestion: Therefore, this is characteristic can be used for prevention, before paraoncogen mutation occurs, in order to prevent cancer creation. After finding the increased breast density, AMF should be eliminated from the patient's living areas so that cancer cannot be created.

\subsection{Sunbathing and Skin Cancer}

Sunbathing increases skin cancer diseases, so people are adviced to avoid sunbathing. This is not true because during sunbathing skin gets red because it is heated and its temperature increases. With skin burned in this way, person goes to bed with AMF. After a longer staying there, skin gets less inflamed and then, there appears body viscous thermo-remanent magnetisation (TVBRM) [3] stronger than BVM, which is the first condition for skin cancer appearance. Gene mutation is possible, and there is the second condition, then Neo can appear. Sunbathing only accelerated TVBRM creation. Sunbathing is necessary because of D vitamin in organism and for human reproduction because it enhances sexual potential. It can be concluded that we should insist on AMF elimination, but sunbath as much as possible.

\subsection{Temperature and Cancer}

Materials-substances with paramagnetic and ferromagnetic characteristics are oriented in magnetic fields. Temperature is opposing to this. It is found that DNA in space has a form of double spiral (helix-like structure). These two spirals can be separated by a cautious heating and connected again by cooling. This is a clear proof that DNA chains are tied by weak magnetic and electromagnetic forces. It is a known fact that tumor tissues are sensible to temperature. There are many works about hyperthermia as a therapy, but results are unexplained and unclear. It is thought that the most important is the space where therapy is applied. The therapy must be applied only in natural EMF. If not, BVTRM will be created and Neo will advance faster. 


\subsection{Genetic Predispositions}

Official medical science mainly connects Neo appearance (CVD, mental disturbances etc.) with genetics. The explanation is that in older ages inclination to getting these diseases is not possible to inherit, but habit to live in enormous AMF can be created. These living spaces pollutants are characteristic for all residential spaces. When a person gets used to living in AMF then she or he, unconsciously, searches this polluted space to stay in. Many experiments show this clearly.

\subsection{Cell's Natural Death Explanation}

Chromosomes magnetise and demagnetise during division [1]. Magnetisation and demagnetisation possibilities are limited for all substances. It is known that telomeres (chromatides' ends) with larger divisions number (cca 50) become thinner and shorter (they peel off) [12], therefore, they cannot magnetise easily because inter-molecular magnetic forces are weaker, shorter, so a cell ends its life cycle.

\subsection{Eternally Living Malignant Cell}

Eternally living malignant cell is created by mutation only in AMF. Malignant cells' telomeres, already created by mutation, can regenerate by new paramagnetic molecules because external surrounding Az, present in tissue, concentrates, i.e., produces enzymes and proteins with increased magnetic characteristics, BVM is formed [3] [4]. In that area, cancerogenous substances marked as electrophiles accumulate [13], i.e., paramagnetics, in science they are called chemical causes of Neo [1] [9]. This means that telomeres' ends, after a lot of magnetisations and demagnetisations, loose magnetic properties, so they are replaced by new paramagnetic substances, this is constantly happening which explains why the cell divides a limitless number times, that is Neo.

\subsection{Low Frequency Electromagnetic Fields Help Fighting Cancer}

By the end of 2011, a group of scientists from USA, France, Switzerland and Brasil published results about "Treating the Advanced hepatocellular Carcinoma with Low Amplitude of Modulated Electromagnetic Fields" [14], in "British Journal of Cancer". The treatment is based on tumor exposing to low frequency electromagnetic waves. In the published work it is not explained how and why this new therapy method seems to be successful as anti-tumor treatment of the patients with advanced hepatocellular carcinoma. There is a brief explanation why is this method applicable in treatment of all MD. This therapy is applicable because of the reason which clearly confirms the cancer cause. As it is known, tumor occurs after forming BVM of an organ or its part, then paraoncogene into oncogene mutation occurs and tumor progressive growth. The BVM formed is the surrounding with high magnetisation, and only in such surrounding cancer cells advance, i.e., divide limitlessly, spreading around on the account of normal tissue. To demagnetise some material, frequent electromagnetic fields must be applied, which force elementary micro-magnetic domains to disorient, i.e., elementary micro-magnetic vectors get chaotic directions. The result is a destroyed cancer tissue BVM. In this environment malignant cells do not function and natural cells spread on the account of tumor tissue. Therefore, the researchers must perform the therapy only in natural EMF, which has been explained in the previous sections. Only in this way the research results will be valid and therapy will be more successful.

Conclusion is suggestion to place every patient into an area with no Az. With the time spent in such areas all BVM will disappear from body. Therefore, AMF measurements in living spaces of diseased are necessary, because only placing the diseased in the natural EMF the post-operative recovery leads to total recovery.

\subsection{Literature Shows That Cancer Behaviour during Various Therapeutic Methods Application Is Unpredictable}

First if all, BVM occurs in an organ or part of an organ BVM occurs, then paraoncogene mutates into oncogene and cancer develops progressively. By the cancer tissue surgical elimination, it is usually impossible to fully eliminate BVM. That is why some cancer types recur quickly, some slowly, and some get eliminated, i.e., patients get healed. It all depends on the occurred BVM size and number, as well as on the phase in which surgical operation is performed on the tissue affected by cancer. Unpredictable behaviour is, also, caused by a deliberate or incidental change of the patient's living space, what can, even, cause full self-recovery. This is, usually, ex- 
plained by some given therapies (various diets, teas, shark cartilage, the living space changed because of geopathogenic radiations etc.), what has nothing to do with the recovery, what is, often, a great delusion of the patient and those who suggest it.

Literature reported that, sometimes, the same type of cancer behaviour upon various therapies application is unpredictable, which is bringing much confusion to oncologists. If the above presented is understood, than it is clear why is this so. Therefore, the diseased person is most often in the areas with various AMF and that is why the same cancer type response to various therapeutic methods is various.

At the end of this section, the necessity of surgical operation of advanced tumors must be pointed out because as larger as possible mass, affected by the disease, must be eliminated, because every BVM depends on the size, i.e., on the mass affected by unnatural magnetization. Then, the diseased should be placed in a natural magnetic field and apply chemotherapy as a therapeutic procedure. Hyperthermia should be applied, mostly, on the body parts from which it is not possible to fully eliminate tumor. With this therapeutic method number of deaths caused by this disease will be reduced.

Therefore, the best prevention for the large number of diseases is to live in natural EMF.

\subsection{Large Number of People Spends Time in High AMF but Small Number Gets Diseased}

Large number of people spends time in high AMF but small number gets diseased... Why? The explanation is that during the night sleep, a part of body located in AMF forms BVM, for example, breast. After few hours of sleeping, person gets up and does everyday work. While moving and working out of bed, BVM degrades, what can be repeated by days, months and years not creating Neo. Then, who gets diseased? The person who, while working (for instance on computer, some machine or in kitchen etc.), enters into AMF again with a breast, continuing to support BVM or even making it stronger. During the night, goes to the same bed with AMF, again, BVM gets stronger and spreads, this repeated by days, months and years makes conditions optimal for Neo appearance. This breast example can be applied to other organs. The conclusion is, if one organ is placed, during working, day and night rest in AMF, relatively quickly, BVM will be created. Paraoncogene mutation occurs during sleeping because body is not much moved from one place, so oncogene, that gives the clonal origin of cancer, forces a cell to divide endlessly and it is malignant cell.

\section{Discussion}

This section will present the issues in research on the cause of cancer. The presented examples are selected from the multitude of cases of AMF measurements in beds in which patients spent night and day. The cause of the cancer was very quickly discovered, by studying the etiological facts and knowing the situation and propagation of AMF in peoples' residential areas. The problem was to force the patient to leave the pathological place of residence because of a habit to enter the present AMF, subconsciously, by inertia. There are experimental evidence of patients entering the AMF and do not notice that they are in it. This is very difficult problem for researchers. Medical condition of a patient staying in natural EMF, was monitored, in order to show complete research and its results. The authors published the results parallel with research, first at local and later on the European and World congresses, conferences and counsel of professional people. The results were spectacular. After long years of research there was a problem appeared by official science and people in government (ministers of health, ecology, etc.). They prevented spreading the research results among the people. They have also banned the media from publishing the results presented in the above mentioned scientific meetings, and to show works that were selected by the Scientific Committee. Those problems are still present.

The authors believe that reason for such behavior is the inertia in heads of authority. For whole life they were convinced that, for example, cancer is influenced by numerous risk factors, and those are the facts remembered in the minds of the people with the potential of $100 \mathrm{mV}$ (millivolts). Information which documents that the only cause of cancer is AMF from the environment, is received in the brains with the potential of $1-3 \mathrm{mV}$. To raise the potential of such newly received information, those people need time, their own experience in measuring of AMF and monitoring patience in natural EMF, and to understand explanations of etiopathological facts.

Those facts point that statistically determined risk factors are explained. It is also been said that there was a misinterpretation of cause and effect. It should be noted that cause of each cancer are AMF. Studies that have persisted for more than 25 years present evidence of the effects of AMF on a cancer i.e. different types which means they are practically the same for all forms regardless of whether they are initiated into different parts of 
the body. When we talk about the location of any cancer (breast, colon, rectum, prostate, lung), the cause is the same, i.e. AMF with variations. From everything presented there is a need to eliminate the AMF from living spaces which will be the greatest help to the patient and the physician in treatment.

\section{Conclusions}

Connection between anomalous zone and body region diseased by cancer is confirmed. Observing the health condition of the malignant diseased patients after moving away from anomalous zone and staying in natural Earth magnetic field, there are no relapses. Explanations of etiological obscurities are complementary with the previous, therefore, it can be concluded: Malignant diseases causes are magnetic field anomalous zones in the spaces where people spend their day and night rest.

For better therapeutic results the most important thing is to move the patient away from anomalous zone and locate in the natural earth magnetic field. Low frequency electromagnetic field application, as a therapy, must be performed only in natural Earth magnetic field. Hyperthermia application, as therapeutic method for neoplasms where cancer has not been surgically completely eliminated, should be performed only in natural Earth magnetic field.

\section{References}

[1] Trifunovic, N. and Cizmic, V. (2014) Breathing Enables the Magnetic Properties of Erythrocytes (HEM Fe) Oxygen, Cells and Carbon dioxide. Journal of Health Science, 2, 270-283.

[2] Catalogue Classification.

[3] Nedeljković, I.S., Kanjuh, V. and Vukotić, M. (1994) “Cardiology”, Belgrade.

[4] Trifunović, N. (1998) Contribution to Knowing of Earth Magnetic Fields Enormous Intensities in the Etiopathogenesis of Cardiovascular Diseases, I Simposium of Cardiovascular Hospitals of Serbia with International Participation-News from Cardiology, Sombor.

[5] Djordjević, M. (1989) Epidemiology of Breast Cancer. Jugoslavija publik, Beograd.

[6] Suša, S. (1979) Endemic Nephritis. Savremena administracija, Belgrade.

[7] Djurić, D. (1966) Biochemistry and Biophysics of Industrial Poisons. Institute for Professional Qualification of Health Workers of SRS, Note 10, Belgrade.

[8] Dimitrijević, B. (1994) Cancer Civilisation Challenge. Institute for Nuclear Sciences Informer, “Vinča” No. 7, Belgrade.

[9] Diklić, V., Kosanović, M., Dukić, S. and Nikoliš, J. (2001) Biology with Human Genetics. Medical Faculty, Belgrade.

[10] Maksimović, N. and Spanopoulos, K. (2008) Descriptive-Epidemiologic Characteristics of Lung Cancer in Serbia, Medical Review, 6, 16-21. http://dx.doi.org/10.2298/MPNS0802016M

[11] Andjelic, S. and Djakovic, E. (2007) My Right to Be Healthy. Research on Reproductive Women with Invalidity Health, July, Belgrade, Serbia.

[12] Guyton, A. and Hall, J. (2010) Medical Physiology. Savremena Administracija, Crnotrafska 7-9, Belgrade.

[13] Hraboč, B., et al. (1991) Informations about Cancerogens. Novi Sad, Serbia.

[14] (2011) Treating of Advanced Hepathocelular Carcinoma with Low Amplitude Modulated Electro-Magnetic Fields. British Journal of Cancer. 